International Journal of Engineering \& Technology, 7 (3.6) (2018) 330-333
International Journal of Engineering \& Technology
SPC
Website: www.sciencepubco.com/index.php/IJET
Research paper

\title{
Posture Instability of People Diagnosed with Parkinson's Disease-A Review
}

\author{
T. Ravi ${ }^{1 *}$, Rajesh Ranganathan ${ }^{2}$, A. Sivasankar ${ }^{3}$ \\ ${ }^{1}$ Junior Research Fellow, Department Of Mechanical Engineering, Coimbatore Institute Of Technology, Coimbatore, Tamil Nadu, India. \\ ${ }^{2}$ Professor, Department Of Mechanical Engineering, Coimbatore Institute Of Technology, Coimbatore, Tamil Nadu, India. \\ ${ }^{3}$ Junior Research Fellow, Department Of Mechanical Engineering, Coimbatore Institute Of Technology, Coimbatore. \\ *Corresponding Author E-Mail:Travi.Cit@Gmail.Com
}

\begin{abstract}
The intent of the paper is to ascertain the reasons behind the posture instability which is being the most disabling feature encountered by the people diseased with Parkinson's which interns leads to risk of fall thereby increases the fractures with history of falls. In this paper a literature survey has been carried out to look after the recent inventions/aids in the clinical aspect and mechanical aspects for supporting PD patients to walk freely without Fear of Falling(FOF).
\end{abstract}

Keywords: Parkinson's disease, posture instability, balance, stability, FOF.

\section{Introduction}

The second most common neurodegenerative disorder after Alzheimer's disease is the Parkinson's Disease (PD) with a male to female ratio of 3:2 in most studies[1] and affects $1 \%$ of the people over the age of 60 years [2] with a frequency of nearly 250 per 100,000 persons [3] although it can occur with younger people also [4].Nearly 7-10 million people are affected with PD an estimation report revealed by Parkinson's Disease foundation [5]. It primarily originates when dopamine generating ("dopaminergic") neurons gets affected in the part of the brain namely "substantia nigra" as an outcome, dopamine content begins to diminish with the advancement of symptom's. The growth of symptom's will progress gradually over years and generally differ from person to person due to the multiplicity of the disease. Dopamine assist in controlling the movements by delivering the signals to the brain which authorize the muscles act smoothly to accomplish the appropriate task. If a person gets affected with Parkinson's Disease will no longer possess adequate dopamine to govern the movements.

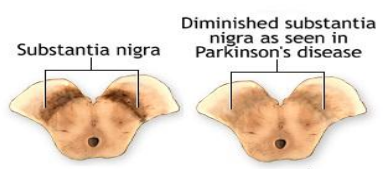

Fig. 1: Patient brain with parkinson's disease[6]

\section{Causes Of Parkinson's Disease}

The root cause of Parkinson's disease is mysterious but some ancestral and environmental factors believably involved, discounting that several factors like pesticide exposure and other toxics, positive family history and oophorectomy have been found for sporadic PD, However the utmost significant one documented so far is the age[1]. Thus, the frequency of disease likely to increase dramatically in the coming decades as the population age which might elevate serious dilemmas at a worldwide level with regard to social security and health care systems which might be mainly true for developing countries such as Chinaor India[7].

\subsection{Primary Symptoms of Parkinson's Disease}

Fig 2. Represents the Primary symptoms of Parkinson's disease which are primarily considered to be affecting the quality of life of these people.

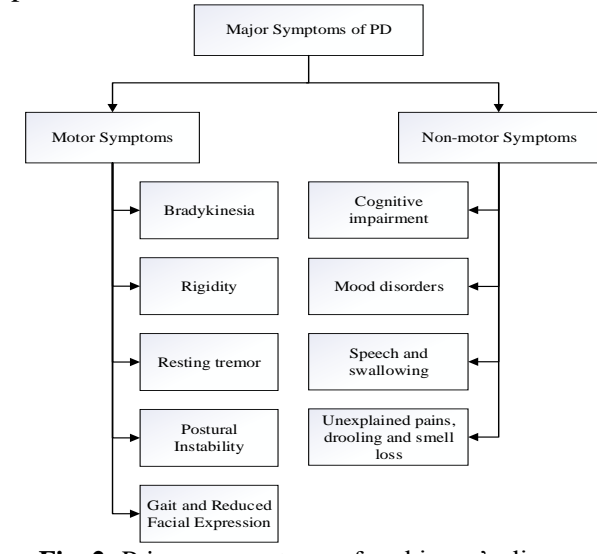

Fig. 2: Primary symptoms of parkinson's disease

\subsubsection{Motor Symptoms}

Motor symptoms or Cardinal symptoms of PD are generally familiar for people as the symptoms can be effortlessly ascertained from outside.

\section{(i). Bradykinesia}

Bradykinesia is a Greek term which means "slow movement ". The symptoms of bradykinesia are shuffling, dragging one or both feet, fewer or no facial expression, freezing of muscles, and difficulties like buttoning clothes, combing hairs, brushing teeth, 
clapping hands and over the time will lose the ability to speak clearly.

\section{(ii). Rigidity (Stiffness)}

Typically, people with Parkinson's Disease experience rigidity in their shoulders, arm, leg muscles. It can also occur in the hips, ankles, neck, trunk and for some people stiff, painful shoulder is the earliest symptoms of Parkinson's disease. Rigidity can occur in the one side of the body (unilateral) or both sides (bilateral). Just like some neurological conditions where muscle tone get affected, here rigidity affects flexor and extensor muscles equally for people with Parkinson's disease. Rigidity causes more stiffness in a downward cycle because it tends to prevent move easily and this will lead to discomfort and pain in muscles.

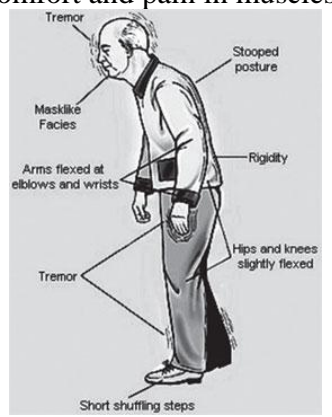

Fig. 3: Sketch of a man with PD primary symptoms(Hutton et al., 2016).

\section{(Iii). Resting Tremor}

Involuntary and rhythmic movements that are associated with one or more parts of the body are called as tremors[9]. Tremors can be differentiated within a movement disorder pathology based on the below listed factors and depending upon the circumstances during which it tends to appear like;(1) at rest, during maintaining certain positions or while voluntary actions are performed; (2) area of the body affected like arms, hands and other parts of the body; (3) the frequency of vibration associated with tremors: low $(<4 \mathrm{~Hz})$, medium $(4-7 \mathrm{~Hz})$ or high $(>7 \mathrm{~Hz})$ frequency bands[4].Most characteristic of PD tremor is the Resting tremor which produces vibration in the frequency range between $4 \mathrm{~Hz}$ to $6 \mathrm{~Hz}$ tends to vanish with a small voluntary movement and for diagnosis of PD its presence is a good criterion because this type of tremor is generally not associated with other pathologies [4].

\section{(iv). Posture Instability}

One of the clinical hall mark of Parkinson's Disease is the Posture Instability and the impairment of balance generally occur during the advanced stages of $\mathrm{PD}[10]$ but for some patients it's a presenting symptom [11] as a result PD patients' willhave problems in maintaining the balance to perform day to day tasks such as walking, turning and standing up from sitting etc., due to this inability to sufficiently balance the body's Centre of mass over the base of support, patients with advanced PD are predisposed to loss of equilibrium and falls[12] in which $45 \%$ of all falls will be in forward direction and nearly $20 \%$ falls directed laterally [13].

\section{(V). Gait and Reduced Facial Expressions}

Gait refers to the way of walking and the regular attention of the gait can reveal the earlier diagnostic suspicions for a number of disorders like cerebral palsy, Rett syndrome and Parkinson's disease (MedicineNet's et at.,2016). Impairments of Gait are classified as Freezing of gait, Shuffling gait and Festinating gait or festination. During the advanced stages of Parkinson's disease individuals experience unique shuffling walk with a stooped position and a reduce or vanished arm swing which tends to make difficult to start walking and making turns, they may also freeze in mid-stride and appear to be falling forward during walking.

One of the motor symptom experienced by the PD patients is the mask-like expression (scientific term 'hypomimia')which is not common for all PD patients or to the similar severity due to this facial movements and animation appears to be less(Downward et al., 2017). There are at least 43 muscles in the face to control the face expressions ranging from happiness to anger which functions very well when the dopamine content is sufficient enough to send signals from brain to the control the muscles movement in face and it's not vice versa for PD patients which leads to challenging task for communication with others and will have negative impact on the relationships.

\subsubsection{Non-Motor Symptoms}

Parkinson's Disease is commonly known as the disease with motor symptoms but people are not aware of the other health issues related Parkinson's Disease which are miscellaneous but jointly called as Non- Motor symptoms(Lazarus et at., 2017). It is significant to understand that these symptoms can also be worrying and disabling than the motor symptoms because of this reason consulting a doctor during the early changes is highly recommended and the changes can be noticed years before they get Parkinson's Disease. Some of these symptoms are briefly discussed in this section.

\section{(I). Cognitive Impairment}

It is the one of the common symptom in PD but not everybody diagnosed with PD experience them and for some people cognitive changes are mild which may have impact on daily functioning Just like bradykinesia "Slow movement" in Motor Symptoms people here struggle with slow thinking and processing information termed as "bradyphrenia"'(Goldman et al., 2017). The most often affected cognitive domains are working memory and attention, executive function and visuospatial function.

\section{(Ii). Mood Disorders}

Mood disorders are the common among the behavioral disorder which can occur during the early and advanced stages of PD as a result quality of life, daily functioning will get affected. The primary mood disorder in PD are anxiety, depression and apathy which affects the PD patients during the progression of disease. Among the three major mood disorders depression is mostly experienced by PD patients with an estimate prevalence rate to be between 2.7 to $90 \%$.

\section{(iii). Speech and Swallowing}

Speech disorder or hypokinetic dysarthria is one of the distressing symptom of PD which occur with $75 \%$ of the PD people and severity of the symptom increases with the advancement of the disease [18]. Disturbance of speech is categorized by monotony of pitch and loudness, imprecise articulation, reduced volume, and a breathy and hoarse vocal quality[19]. Difficulty in swallowing or Dysphagia is a common issue for PD people has consequences like shorter survival time due to difficulty of swallowing which arises due to the affected muscle of the throat hence intake of food decreases which leads to under-nutrition of the patient. It can also result in weight loss, pneumonia, dehydration and airway obstruction.

\section{(iv). Unexplained Pains, Drooling and Smell Loss}

One of the key non-motor symptoms are unexplained pain which are frequently unrecognized and under treated[19] and the number of studies revealed that pain is frequent which is still 
underestimated or under treated non motor symptom affecting nearly $83 \%$ of the people diagnosed with Parkinson's disease[20] and the pains associated with PD are musculoskeletal, dystonic, central and neuropathic pain [20].The reasons for the pain is yet to be ascertained and completely understood because of the adverse effect on the quality of the patients life [21].

Drooling produces lot of negative physical and psychosocial consequences for PD patients and their caregivers due to its symptom like extreme pooling and spillover of saliva out of the oral cavity (Srivanitchapoom and Pandey, 2014).

Smell loss or hyposmia is a reduced loss of smell experienced by PD patients is one of the earliest symptoms which can include impairments in odor detection and losing this sense of smell can have a diverse effect to taste of food also because as these are closely linked. The loss of smell is caused by number of reasons likes allergies, head trauma, viral infections or smoking and hyposmia symptom is common with PD and Alzheimer's disease patients who are unaware of their decreased sense of smell prior to being tested.

\section{Current Trends in Posture Instability}

Out of all the symptoms of Parkinson's disease Posture Instability is perhaps the most dangerous symptom because of the decreased mobility and increased falling rate results in damaging the quality of life of PD patients, So improving the Posture stability can certainly change the quality of life of PD patients therefore here a literature survey is been carried to out to look after the available source of medication if any or aids on the clinical and mechanical aspects which are available for the them to fight against this symptom to live normal life without any dilemmas.

\subsection{Clinical Based Studies of Posture Instability}

Posture Instability precedes to devastating recurrent falls in patients with Parkinson's disease with an occurrence rate of $13 \%$ weekly and $70 \%$ yearly [23] which accelerates with increasing age and severity of the disease. The risk of falls leads to direct consequences like hip fractures, head trauma, contusions and other injuries or even death[24].Recent inventions/ developments and studies regarding the latter is been discussed below.

Posture instability leads to increased risk of falls and cannot be diagnosed by pharmacological therapyso to improve the posture stability Stochastic Resonance Therapy(SRT) was used for the patients in the adavanced stage of the disease for whom physiotherapy is not recommended therefore suggested SRT to be a novel potential treatment which yielded significant results in improving the postural stability even in individuals with high risk of falling[25]. In this study a total of 56 PD members were assigned to either experimental or shamgroups in which four series of vibration were received by experimental group for eight days by using randomized whole body vibration with each series consists of six stimulus trains for a duration of 60 -seconds and below Fig 4 represents the number of patients assesed for diagnosing posture instability through SRT (whole body vibration)[25].

In the old age adults, Fear of falling (FOF) is prevalent [26]its frequent with people with balance issues (PD patients) moreover degree of FOF is unknown in PD [26] conducted a study to find out FOF qualitative and quantitative posture control relationships by study of fifty eight nondemented PD patients along with healthy adults of same age group. The Degree of FOF was estimated using Activities-specific Balance Confidence scale and Unified Parkinson Disease Rating Scale was used to assess the Qualitative posture control in which Posture control was computed for eight standing balance tests of unique challenges through the centre of pressure measurements obtained from a force plate and the results revealed that when comparing to healthy adults the FOF evident was more for PD patients with greater degree of posture impairment.

Pathophysiology of falls is an important thing to be understood for preventing falls in PD patients but the possible fundamental mechanisms of such falls yet to be fairly explained [27]. An investigation was carried out by [27] to forecast the mechanism of recurrent falls based on the falling direction in which $62 \mathrm{PD}$ patients with recurrent falls were divided into two groups according to the direction of falling (45 PD forward fallers and 17 PD Non forward fallers) and the results shown that forward fallers experienced critical falls during turning or walking compared to the non-forward fallers during standing or sitting or turning and freezing of gait (FOG) was higher in case of forward fallers.[27] Demonstrated that from the regression analysis falling forward is associated with FOG and Non forward falling is associated with balance impairment, a kinetic-rigid subtype, and neuropsychiatric symptoms and concluded that balance impairment and FOG are two key accountable variables for mechanism of recurrent falls and falling direction being the important predictor for these mechanisms.

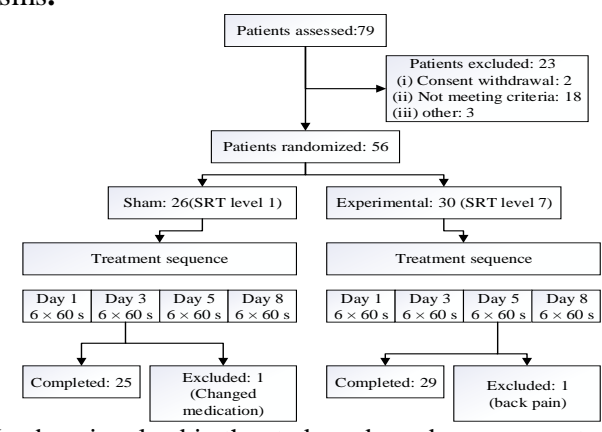

Fig. 4: Members involved in the study and numbers represents the number of patients in each category[26]

\subsection{Recent Developments of PD Walking Aids}

In the mechanical aspect of inventions not much development of walking aids has been seen till date except aids like dinning aids, bathing aids, grooming aids, writing aids etc and very few inventions regarding the mobility aids were brought in to the society for helping PD patients to deal with mobility issue but considering the aesthetic point of view and the economic factors these inventions may or may not satisfy the user. From the internet database search assistive mobility aids available for Parkinson's disease are very few like U-Step II Walking Stabilizer and Walk to Beat.

\subsubsection{U-Step II Walking Stabilizer:}

U-Step II Walking Stabilizer shown in Fig 5 is one of the newest mobility aid developed in the recent times for the people who suffer from neurological issues to eradicate risk of falling and increase independence with lots of feature like more stability, manoeuvrability and user-friendly controls lets the user to feel comfortable because of the ergonomics and well positioned handle bars which ensure to minimize the stress on the back and joints thereby encouraging them to stand upright stance and a laser light line projected towards to the walking path ensures the patient to walk freely without having issue with freezing and gait [28]

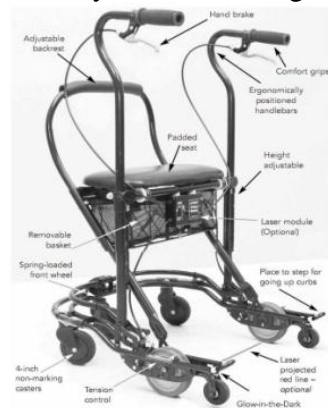

Fig. 5: U-Step II walking stabilizer[29]

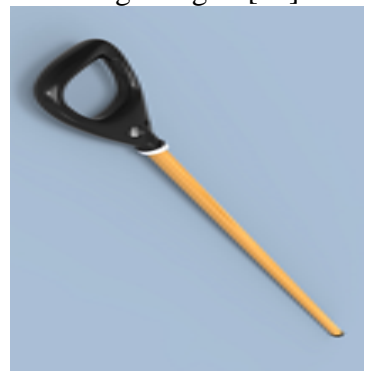

Fig. 6: WalktoBeat[30] 


\subsubsection{WalktoBeat}

Posture instability is being the one of the major issue for walking in addition to this freezing and gait will be experienced by the PD patients when they start walking so considering the issue a device named WalktoBeat shown in Fig 6 [29] developed with the latest technology installed in the handle offers constant rhythm which can only be felt in the handle, not seen or heard considering the patients dignity and emotions. A non-stop indicating beat felt by vibration (with a changeable pace) is a novel approach method to freezing and gait abnormalities.

\section{Conclusion}

The number of case studies lastly revealed that most of the people diagnosed with PD are having a very bad experience with quality of life due to one of the clinical hall mark "Posture instability" in the advanced or earlier stages of PD which increases risk of falls during the life expectancy and direct consequences of falling are fractures, particularly hip fractures, head trauma, contusions and other injuries or even death. The study also revealed that very few aids are available for evading the posture instability. So, a design and development of a device considering the aesthetics and economical factors which can helps the patients to overcome the posture instability will be highly appreciated and will certainly have a high impact in enhancing the PD patient's quality of life.

\section{Acknowledgment}

We gratefully acknowledge the financial support for establishing the Centre of Excellence in Manufacturing Sciences at Coimbatore Institute of Technology, Coimbatore; India from Ministry of Human-Resource Development (MHRD), Govt. of India where the sample/R\&D work has been carried out.

\section{References}

[1] De lau LML \& Breteler MMB, "Epidemiology of Parkinson's disease", J. Neural Transm., Vol.124, No.8, (2017), pp. 901-905.

[2] Behari M, Srivastava AK, Das RR \& Pandey RM, "Risk factors of Parkinson's disease in Indian patients", Journal of the neurological sciences, Vol.190, No.1,(2001), pp.49-55.

[3] Nilashi M, Ibrahim O, Ahmadi H, Shahmoradi L \& Farahmand M, "A hybrid intelligent system for the prediction of Parkinson's Disease progression using machine learning techniques", Biocybern. Biomed. Eng., (2017), pp.1-15.

[4] Camara C, Isasi P, Warwick K, Ruiz V, Aziz T, Stein J \& Bakštein E, "Resting tremor classification and detection in Parkinson's disease patients", Biomedical Signal Processing and Control, Vol.16, (2015), pp.88-97.

[5] Perumal SV and Sankar R, "Gait and tremor assessment for patients with Parkinson's disease using wearable sensors", ICT Express, Vol.2, No.4, (2016), pp.168-174.

[6] Joseph V \& Campellone, "Substantia nigra and Parkinson disease: MedlinePlus Medical Encyclopedia Image”, 2015.

https://medlineplus.gov/ency/imagepages/19515.htm.

[7] Dorsey E, Constantinescu R, Thompson J, Biglan K, Holloway R \& Kieburtz K, "Projected number of people with Parkinson disease in the most poplous natios, 2005 through 2030", Neurology, Vol.68, (2007), pp.384-386.

[8] Hutton T, "Does Hillary Clinton Really Have Parkinson's Disease?", Views From Medicine Spirit Ranch, (2016).

https://tomhuttonmd.com/2016/09/14/does-hillary-clinton-really-haveparkinsons-disease/.

[9] Abdo WF, van de Warrenburg BPC, Burn DJ, Quinn NP \& Bloem BR, "The clinical approach to movement disorders", Nat. Rev. Neurol., Vol.6, No.1, (2010), pp.29-37,

[10] Pillon B, Dubois B, Cusimano G, Bonnet AM, Lhermitte F \& Agid $\mathrm{Y}$, "Does cognitive impairment in Parkinson's disease result from non-dopaminergic lesions?", J. Neurol. Neurosurg. Psychiatry, Vol.52, No.2, (1989), pp.201-206.
[11] Klawans HL \& Topel JL, "Parkinsonism as a falling sickness", JAMA, Vol.230, No.11,(1974), pp.1555-1557.

[12] Morris M, Iansek R, Smithson F \& Huxham F, "Postural instability in Parkinson's disease: A comparison with and without a concurrent task", Gait Posture, Vol.12, No.3, (2000), pp.205-216.

[13] Bloem BR, Hausdorff JM, Visser JE \& Giladi N, "Falls and freezing of Gait in Parkinson's disease: A review of two interconnected, episodic phenomena", Mov. Disord., Vol.19, No.8, (2004), pp.871-884.

[14] MedicineNet's, "Medical Definition of Gait", 2016

https://www.medicinenet.com/script/main/art.asp?articlekey=3533.

[15] Downward E, "Symptoms-Mask-like Expression-Parkinsons Disease.net", (2017).

https://parkinsonsdisease.net/symptoms/hypomimia-mask-likeexpression/.

[16] Lazarus J, "Non-Motor Symptoms”, (2017).

http://www.parkinson.org/Understanding-Parkinsons/Non-MotorSymptoms

[17] Goldman G, “Cognitive Changes”, (2017).

https://www.apdaparkinson.org/what-is-parkinsons/symptoms/ cognitive-changes/.

[18] Theodoros DG, Constantinescu G, Russell TG, Ward EC, Wilson SJ \& Wootton R, "Treating the speech disorder in Parkinson's disease online", J. Telemed. Telecare, Vol.12, (2006), pp.88-91.

[19] Wallace VCJ \& Chaudhuri KR, "Unexplained lower limb pain in Parkinson's disease: A phenotypic variant of 'painful Parkinson's disease", Park. Relat. Disord., Vol.20, No.1, (2014), pp.122-124.

[20] Wasner G \& Deuschl G, "Pains in Parkinson disease-many syndromes under one umbrella", Nat. Rev. Neurol., Vol.8, No.5, (2012), pp.284-294.

[21] Martinez-Martin P, "The importance of non-motor disturbances to quality of life in Parkinson's disease", J. Neurol. Sci., Vol.310, No.1-2, (2011), pp.12-16.

[22] Srivanitchapoom P, Pandey S \& Hallett M, "Drooling in Parkinson's disease: A review", Park. Relat. Disord., Vol.20, No.11, (2014), pp.1109-1118.

[23] Farombi TH, Owolabi MO \& Ogunniyi A, "Falls and Their Associated Risks in Parkinson's Disease Patients in Nigeria", Journal of movement disorders, Vol.9, No.3, pp.160-165.

[24] Contreras A \& Grandas F, "Risk of falls in Parkinson's disease: A cross-sectional study of 160 patients", Parkinsons. Dis., (2012).

[25] Kaut O, Brenig D, Marek M, Allert N \& Wüllner U, "Postural Stability in Parkinson's Disease Patients Is Improved after Stochastic Resonance Therapy", Parkinsons. Dis., (2016).

[26] Adkin AL, Frank JS \& Jog MS, "Fear of falling and postural control in Parkinson's disease", Movement Disorders, Vol.18, No.5,(2003), pp.496-502.

[27] Youn J, Okuma Y, Hwang M, Kim D \& Cho JW, "Falling Direction can Predict the Mechanism of Recurrent Falls in Advanced Parkinson's Disease", Sci. Rep., Vol.7, No.1, (2017), pp.7-12.

[28] Jonathan M, “The U-Step II Walking Stabilizer”, 2017.

http://www.ustep.com/images/ustep2br.pdf.

[29] Neha C, "Walk to Beat", 2017.

http://walktobeat.co.uk/index.html. 